\title{
The Investigation of Anti-Jamming Technology For Temperature Measure System in Spacecraft Thermal Test
}

\author{
An Wanqing, Zhu Xi, Feng Yao, Liang Shuo and Wang Qingyu \\ Beijing Institute of Spacecraft Environment Engineering, YouYi Road, Beijing, China
}

\begin{abstract}
At present, the spacecraft thermal test temperature measurement system has not been designed effectively. In the spacecraft experiment, the defects of anti-interference ability and system instability are exposed. Firstly, the composition and status of the temperature measurement system of spacecraft thermal test are analyzed. And then we set up a system to interfere with fault tree according to existing problems, locate the problem and determine the source of the interference. Finally, the anti-interference scheme and implementation are developed, and the test data of anti-interference test system is set up. The anti - jamming performance of the anti - jamming scheme of thermal test temperature acquisition system was evaluated. The results show that it can improve the anti-jamming capability of the spacecraft thermal test temperature acquisition system and improve the stability of the system.
\end{abstract}

\section{INTRODUCTION}

Vacuum thermal test is an indispensable project in the development of spacecraft; Temperature measurement and control in the experiment have a direct on the test results. Therefore, the correctness and validity of temperature data measurement is the key to the success or failure of spacecraft vacuum thermal test.

With the development of space technology, the equipment and tooling involved in thermal testing is increasing complex. High power motor and mobile mechanism are widely used in the experiment, the problem of signal interference is followed by the abnormal beating of thermocouple temperature test data in several experiments, which has serious impact on the thermal test ${ }^{[1]}$. The interference source is not easy to determine, the interference form cannot be judged, and the interference cannot be effectively suppressed.

In order to guarantee the accuracy of measurement, analyse the causes of interference, the interference sources 、 the interference form, improve the antiinterference ability of temperature measuring system. Firstly the present situation and composition of temperature measurement system are analyzed in this paper, the cause of interference and the interference sources are determined. And then we develop antijamming solution, the feasibility of the solution is verified in the vacuum thermal test. At last the solutions proposed we choose in this paper can solve the problems existing in the spacecraft thermal test temperature measurement system.

\section{SYSTEM CURRENT STATUS}

\subsection{The status of temperature measurement system}

At present, the spacecraft thermal test temperature measurement system, the voltage or resistance of the temperature sensor on the spacecraft is collected by data acquisition instrument, such as (Keighley3706 、

Agilent4411、Keighley2750 data acquisition instrument). The temperature reference device is placed inside the container, the thermocouple used on the spacecraft is the same as in the reference device. The voltage or resistance signal on the spacecraft is transmitted to the container by a single wire lead, the signal on the measured cable leads to data acquisition instrument. The data acquisition instrument is connected to the computer through Ethernet, the data acquisition program on the computer is written by VB program. The measuring program converts the voltage signal to the corresponding temperature value according to the corresponding scale. All the dates of temperature are stored in the local computer and server's hard disk in real time to form a temperature measurement data file.

The thermocouple lead length of the spacecraft surface is $3-5 \mathrm{~m}$, welding with electrical connector. The lead wire is coated with aluminum foil for protection. The utility line of a thermocouple is placed in a vacuum container in the form of a bare wire. In the container, the transition cable and the external transition cable are

* Corresponding author: Wanqing5010@126.com 
introduced into the measuring instrument access end. No shielding layer is installed in the transition cable in the container.

The current temperature measurement system has no effective anti-electromagnetic interference design, and the system has exposed the disadvantages of poor antiinterference ability and instability during the multispacecraft thermal test. In order to ensure the accuracy of data collection, there are two main techniques: improve the sensitivity of the sensor and improve the antijamming technology of the instrument. With the development of temperature measurement technology, measuring sensor sensitivity is no longer the main factor that impedes the improvement of system accuracy. Therefore, the anti-jamming ability of spacecraft thermal test temperature measurement system is strictly controlled.

\subsection{Composition of temperature measurement system}

The spacecraft thermal test data acquisition system consists of measuring software 、 measuring instrument measuring cable and temperature measuring sensor. The system composition is shown in figure 1 as below.

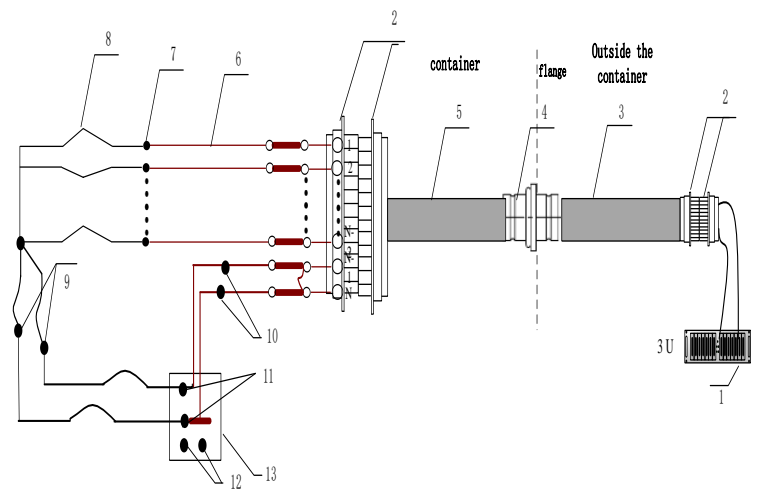

1--Data acquisition instrument; 2-Electric connector; 3--External cable; 4-middle Electric connector; 5Internal cable; 6 -Copper wire; 7--Temperature measuring points; 8-Constantan wire; 9-Constantan wire welding point; 10-Copper wire welding point; 11--Reference point; 12-Platinum resistance; 13--equipment

Figure 1. the composition of temperature measurement system in vacuum thermal test.

\section{SYSTEM ANALYSIS SOURCE DETERMINATION}

\subsection{Eelectromagnetic interference}

Electromagnetic interference mainly refers to radiation interference and conduction interference.

In order to suppress the electromagnetic interference, we need to find the possible source of interference and coupling first, and take effective technical means: including suppressing the interference source 、 suppressing or eliminating coupling. The main technical is grounding 、 shielding and coupling three technologies The thermocouple is used as the temperature sensor in the spacecraft thermal test, and its electromagnetic interference mainly has three points:

(1) Spacecraft magnetic field inside the equipment: The strong current of the equipment in the spacecraft may produce a strong magnetic field. If the temperature sensor compensates the conductor and the spacecraft is close to each other, it will be impressed by the magnetic field. Electromagnetic interference is formed when the input circuit induces the potential.

(2) Welding temperature sensor coupling: The thermal test temperature sensor for spacecraft is thermocouple. The measurement principle of thermocouple is not only the connection of two kinds of materials. When the temperature of the two materials is different, thermoelectric potential is generated in the thermocouple welding and compensation. ${ }^{[3]}$.

(3) Ground interference coupling: refers to the signal grounding, the ground interference including the common line interference grounding, ground loop interference and so on will introduce electromagnetic interference. It is ideal for temperature measuring instruments to be directly connected to the earth or a reference potential to the ground.

\subsection{Power supply system interference}

The causes of dc interference are as follows:

(1) The power supply network produces harmonic interference to the data acquisition instrument. Power supply, power transmission and distribution system, UPS, power equipment access and other multiple reasons can lead to high harmonics in the power grid, which will affect the final test results.

(2) The thermocouple is in contact with the high voltage dc power supply. There is a leakage resistance between the high voltage dc power supply and the measuring system of the instrument when the thermocouple is welding and measuring the temperature with the metal object of the high voltage direct current. The leakage current is interfered by the measurement system. ${ }^{[4]}$.

\subsection{Fault tree of thermocouple temperature measurement system}

Based on the above possible interference reasons and the composition of the thermal test temperature measurement system, the disturbance failure tree of the spacecraft thermal test temperature measurement system is summarized as shown in fig. 2 below. 


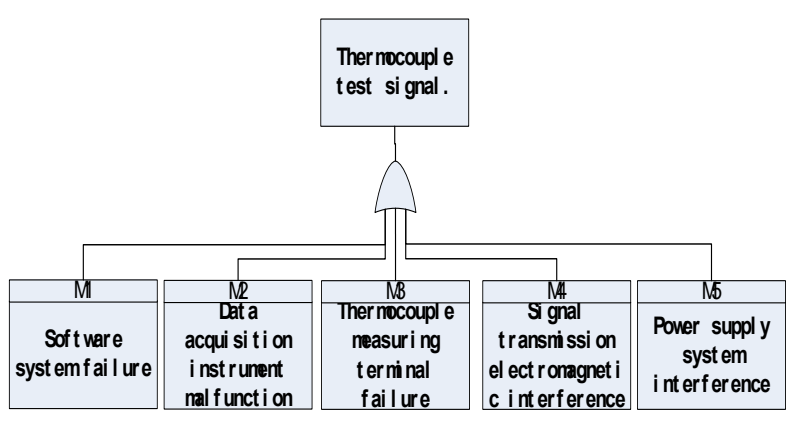

Figure 2. interference fault tree of temperature measurement system

The analysis of the data that has produced the interference results in the conclusion:

(1) M1 (software system failure): During the test, if the software system fails, it may cause a change in the thermocouple measurement data. In several experiments with interference, the software was used multiple times in each system level and component test. So we can eliminate the problem.

(2) M2 (number of equipment failure) : In the process of testing, if the number of instruments fail, the thermocouple measurement data will be changed. In several interference experiment, thermocouple measurement system using data acquisition instrument for 37064411 data acquisition instrument, data acquisition instrument, the two kinds of test instrument in use for many times, no abnormalities are found. After the abnormal occurrence, the data still beats after replacing the instrument. So we can eliminate the problem.

(3) M3 (thermocouple measurement end failure) : If the measuring end of the thermocouple is not well insulated with the test piece, when the surface of the spacecraft has an electric current or a potential difference, the measurement system will be introduced to cause the change of the thermocouple test data. When the thermocouple is pasted on the surface of the spacecraft, it is required to paste the $3 \mathrm{M}$ tape at the designated position and paste the thermocouple measurement end into $3 \mathrm{M}$ tape to insulate the specimen and the thermocouple. Inspection found no damage to the $3 \mathrm{M}$ tape insulation on the surface of the spacecraft. So we can eliminate the problem.

(4) M4 (signal transmission emit): The existing thermocouple is introduced into the data acquisition instrument by the transition cable inside the container. At present, the transitional cable in the container has the shielding layer, and the shielding layer is connected with the electric connector shell. The outer cable of the container has no shielding layer, so the possibility of electromagnetic interference in signal transmission cannot be ruled out.

(5) M5 (power supply system interference): The power supply of the temperature measurement system is disturbed to produce harmonics. The harmonic component reaches the data acquisition instrument through the power supply network, which affects the normal work of the temperature measurement system, and shows that the test data fluctuates with the harmonic frequency. The generation of harmonic generation mainly comes from three aspects, 1 is the power supply quality is not high to produce harmonics; 2. Harmonic generation of power transmission and distribution system; 3 it is the electric equipment that produces harmonics. The current temperature measurement system can not be excluded from the above three aspects.UPS may produce harmonics using the temperature measurement system. Power transformers in power transmission and distribution systems may produce harmonics. In the experiment, the power devices such as the inclination adjustment mechanism and stepping motor can also cause harmonics to the power grid. The change in the unbalanced current may also be the change of the power grid waveform. Therefore, the possibility of interference of power supply system cannot be ruled out.

\subsection{Determination of interference source}

According to the research on thermocouple measurement system. The interference signal of thermocouple measurement system is divided into common mode interference and serial mode interference. The interference form can be divided into electromagnetic interference and harmonic interference. Harmonic disturbance is caused by nonlinear load in power supply network. When the current flows through the load, it does not have a linear relationship with the voltage added, forming a non-sinusoidal current. Therefore, harmonic interference is generated in the measurement system.

Serial mode interference is the interference of the data acquisition instrument due to the communication signal between the input terminals of the data acquisition instrument. The interference voltage is about a few mill volts to a few tens of mill volts, and the principle of interference is shown in figure 3 .

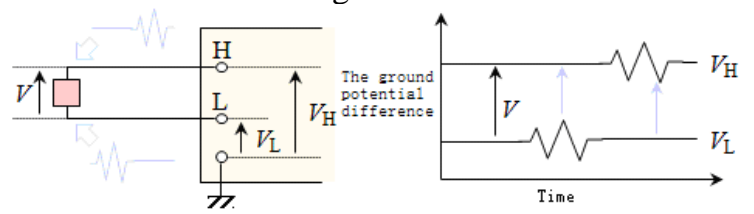

Figure 3. schematic diagram of serial mode interference

There are several types of serial mode interference:

(1) Alternating magnetic field: The power transformer, ac motor, strong current wire and so on have strong alternating magnetic field. If the compensation wire is passed in the vicinity it will be affected by the alternating magnetic field. The ac potential is induced in the input loop to form interference;

(2) The interference of the thermocouple welding on the electrified body: in the case of some special requirements, the working end of the thermocouple should be pasted on the surface of the metal material. The interference voltage is introduced because of the potential difference of the metal specimen in the direction of the current direction.

(3) The string mode interference signal is caused by the voltage drop caused by the interference current through the thermocouple and its connection wire or the impedance of the measuring system ${ }^{[5]}$. 


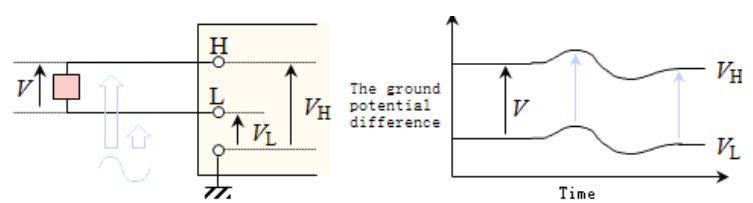

Figure 4. schematic diagram of common mode interference

Common mode interference is usually caused by the difference between the data acquisition instrument and the temperature sensor. The interference voltage can be ac voltage or dc voltage. The maximum amplitude can be up to several volts according to the grounding condition and the field condition. The interference principle is shown in figure 4.

C Common mode interference is generated: in the spacecraft thermal test, the number of temperature sensors on the star is large and dispersed in different locations. A longer thermocouple line is used to transmit the signals sent by the sensor to the data acquisition instrument. Data acquisition instrument ground point with the contact of the temperature sensor location there is a potential difference between $\mathrm{Ua}$, data acquisition instrument is the two input end of the $\mathrm{AD}$ converter, respectively, have $U$ and $U+U a$ two signals, $U a$ is the common-mode interference voltage converter ${ }^{[6]}$, the principle is shown in figure 5.

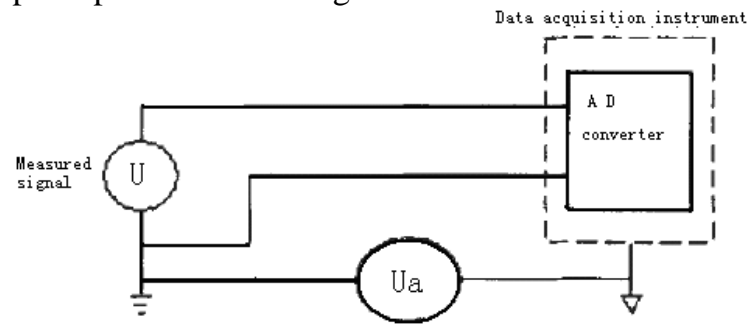

Figure 5. schematic diagram of common mode interference of spacecraft thermal test

\section{$4 \quad$ ANTI-INTERFERENCE FORMULATION AND VERIFICATION}

SCHEME TEST

\subsection{System anti-interference scheme formulation.n}

The time domain analysis is conducted for thermocouple measurement data. During the analysis of the temperature data changes, the similarity and correlation of the thermocouple measurement signals at different moments were obtained. The time frequency analysis of the thermocouple measurement data is made through the phase plane of two coordinates of the time axis and the frequency axis. The frequency composition of the whole thermocouple signal in local time domain is obtained. Or we can see the distribution and arrangement of the whole thermocouple signal in local time. The interference signal components are identified as signal transmission and power supply system interference. The following antiinterference measures are formulated:
(1) Temperature sensor signal line shielding measures: use metal shielding layer to wrap the temperature sensor signal line. The flux will pass through the metal shielding layer, which is very small, so the effect on the signal line will be reduced;

(2) Temperature sensor and other potential shielding: the shielding layer of the temperature sensor is not grounded and is connected to the negative terminal or positive terminal of the sensor. The shielding layer is equal to the potential of the measurement system, and there is no potential difference between them. The leakage current will be passed along the shielding layer and the circuit of the distributed capacitance to the shielded conductor;

(3) A filter is added to the signal input end of the temperature measurement system, and the appropriate filter is selected to carry out the implementation according to the interference signal spectrum determined by the analysis.

(4) Install the isolation transformer at the power end of the temperature measuring system. The disturbance caused by isolating the power supply system.

\subsection{Test verification of anti-interference scheme}

This test is carried out in the spacecraft space environment simulator. Simulate the real environment of spacecraft thermal test. The high power air conditioning system is connected to the temperature measurement system as the interference source. The dynamic interference of the temperature measurement system in the work of air-conditioning system is evaluated. The specific test steps are as follows:

(1) On the surface of the spacecraft, 44 thermocouple thermometers were set as temperature monitoring, and two platinum resistance temperature points were used as cold end compensation;

(2) Using two Agilent 4411 measuring instruments as temperature acquisition equipment. One of them shares the same power and ground terminal with the power supply, and the other is a separate power supply and earthling terminal;

(3) Reduce the surface temperature of the spacecraft to the lowest temperature of the environmental simulator. Turn on the high power air conditioner and observe the temperature change of thermocouple on two temperature acquisition equipment. The data changes are shown in figure 6 and 7.

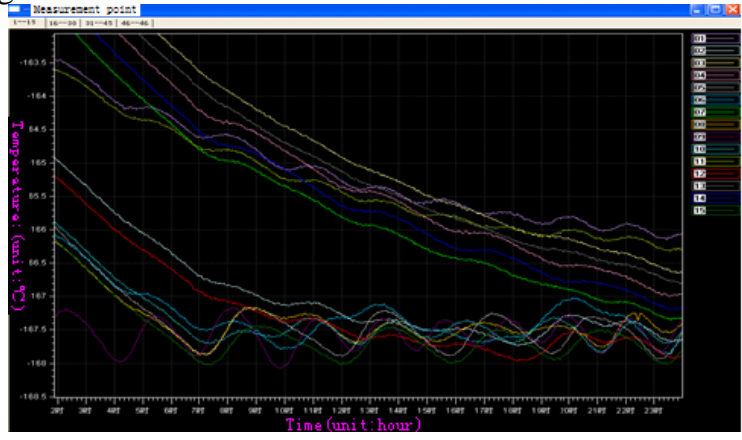

Figure 6. the temperature control curve before interference 


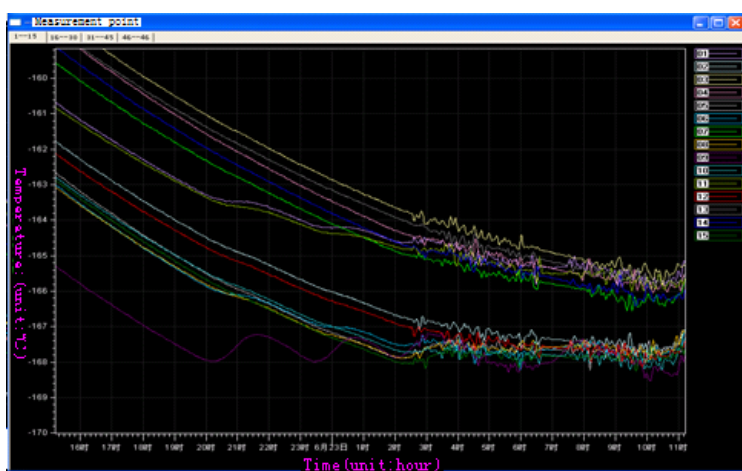

Figure 7. the temperature control curve after the interference is opened

(4) In the same condition, the anti-interference device isolation transformer was added before the first temperature acquisition device. The change curve of thermocouple temperature on the test piece is shown in figure 8 .

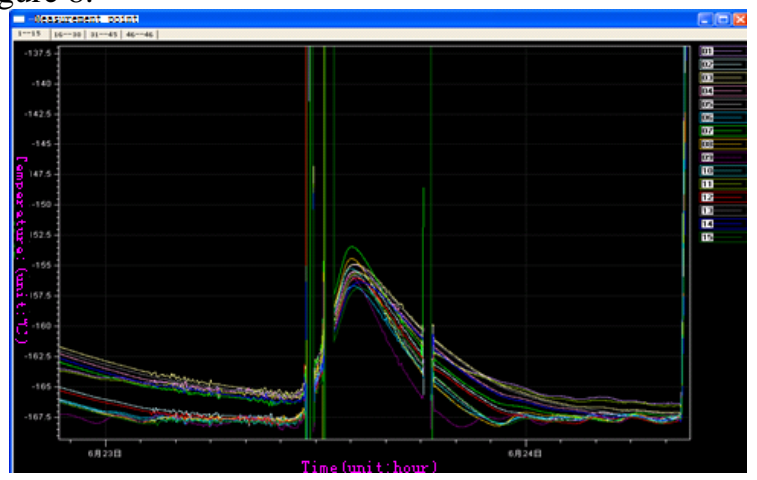

Figure 8. increases the temperature change curve after isolating transformer

Test result: when the high-power air conditioning works, the harmonic feedback will be generated on the power grid, which will affect the thermal test temperature measurement system. The measurement error can directly affect the accuracy of the measurement results by measuring the instrument directly to the measuring end of the thermocouple. At the front end of the measuring equipment power supply system, the isolation transformer can be used to filter the high harmonics of the load, reduce the high frequency interference, and reduce the high harmonics greatly.

\section{CONCLUSIONS}

This paper introduces the composition and status of spacecraft temperature measurement system. Firstly, we analyze the existing system interference, and determine the interference source and anti-interference measures. The following problems and corresponding improvement methods can be determined in the system. Increasing APF and adding isolation transformer at the front end of the measuring equipment can effectively reduce the interference of common and differential mode voltages. Adopting shielding technology and grounding technology to suppress the interference source. It is effectively reduce system interference.

\section{REFERENCES}

1. Huang bencheng, ma courtesy. Spacecraft space environment test technology [M]. Beijing: national defences industry press, 2001: 7-8.

2. Zhang jingchuan et al. Application status and development trend of spacecraft vacuum thermal test and control system $[\mathrm{J}]$. Spacecraft environmental engineering,2012,29(3):263-267.

3. Jiang zhu et al. A design and implementation of a temperature signal isolation transmitter with high anti-interference ability $[\mathrm{J}]$. Space measurement technology,2016,36(2):2-5.

4. Wei yongfan. The anti-interference problem of thermocouple measurement temperature [J]. Mechanical and electrical information,2011,6(6):3236.

5. Niu jianbo et al. Research on anti-interference technology of resistance temperature measurement [J]. Electronic world.2013,8(24):116-119.

6. Ma weidong. Anti-interference measures of thermocouple [J]. Cement.2011,4(3):39-42. 